\title{
THE ENGULFING THEOREM FOR LOCALLY TAME SETS
}

\author{
BY M. H. A. NEWMAN
}

Communicated by O. G. Harrold, April 13, 1966

1. The theory of combinatorial manifolds, now some forty years old, has been strikingly successful recently in solving problems of homeomorphism and isotopy, but in spite of recent improvements, notably by Zeeman [3], it remains a difficult medium to work in, cut off by its nature from the ordinary methods of topology.

The work here reported on is a first application of a different method which, while keeping to the spirit of Whitehead's "expanding" and "collapsing", stays nearer to topological notions, by substituting linear isotopies for addition and subtraction of simplexes. The Stallings engulfing theorem is proved in the following form.

Given a locally tame closed set, $X$, of dimension $p \leqq n-3$ in a $p$ connected topological n-manifold-without-boundary, $M$, and a ( $p-1)$ connected open set, $V$, of $M$ such that $X \backslash V$ is compact, there is a homeomorphism, $h: M \simeq M$ such that $X \subseteq h V$.

$X$ is called "locally tame" in $M$ if for every $x \in X$ there is a neighbourhood $U(x)$ in $M$ such that $X \cap \bar{U}$ is a complex-locus (by the standards of a coordinate system in $\bar{U}$ ). In fact the theorem is proved for the larger class of $p$-dominated sets, $p \leqq n-3$, such that $X \cap \bar{U}$ is contained in a $p$-complex in $\vec{U}$.

The homeomorphism $h$ is shown to be isotopic to 1 and is 1 outside a compact set. It is composed of a finite number of small "pushes", each of which moves only a set of points in a euclidean neighbourhood $\bar{U}$ along parallel lines in $\bar{U}$.

The general scheme of the proof is that of Stallings [2], but the details are different.

E. H. Connell has shown, using Stallings' stretching process between dual subcomplexes [1], that this form of the engulfing theorem implies the Poincaré Hypothesis for topological manifolds $(n \geqq 5)$.

2. The theorems used in place of the regular-neighbourhood theory are concerned with real semicontinuous functions on a metric space, ${ }^{1}$ $X$. If $f, g$ are two such functions with $f \leqq g$, and if $A \subseteq X$, the prism $\Pi(f, g \mid A)$ in $X \times R(R$ the real line $)$ is $\{(x, t) \mid f .(x) \leqq t \leqq g(x, x) \in A\}$, where $f$. and $f \cdot$ are $\lim \inf f$ and $\lim \sup f$. We put $\Pi(f, f \mid A)=\Pi(f \mid A)$. (By admitting semicontinuous functions the theory can deal with the

${ }^{1}$ Or any paracompact space, if a few extra precautions are taken. 
prisms in simplexes, etc. that occur in the engulfing theorems.) The basic tool is the theorem of Baire which says that if $f \leqq g$ and $f$ is u.s.c., $g$ is 1.s.c., then there is a continuous function $\phi$ satisfying $f \leqq \phi \leqq g$.

The simplest engulfing theorem is the

LEMMA. Let $f_{0}, f_{1}$ be continuous functions on the closed set $F$ in $Y$, with $f_{0} \leqq f_{1}, f_{0}=f_{1}$ on $\operatorname{fr}_{Y} F$; and let $U, W$ be open in $Y \times R$ with

$$
\Pi\left(f_{0}, f_{1} \mid F\right) \subseteq W, \quad \prod\left(f_{0} \mid F\right) \subseteq U .
$$

Then $\Pi\left(f_{0}, f_{1} \mid F\right)$ can be engulfed by $h U$, where $h$ is active only in $W$.

The conclusion means, besides $\Pi\left(f_{0}, f_{1} \mid F\right) \subseteq h U$, that $h \cong 1$ by an isotopy which is 1 outside $W$. In fact $h$ is a p.l. homeomorphism of each line $x \times R$ on to itself.

To be useful the Lemma must be strengthened by allowing $f_{0}$ to be u.s.c.; the space to have an extra factor, $X \times Y \times R$, so as to be applicable to the engulfing of a set in a $q$-simplex in $M^{n}$; and provision must be made for retaining in $h U$ a set already contained in $U$. This is provided for in

THEOREM A. Let $g_{0}, g_{1}$ be functions on the closed set $F$ in $Y$, with $g_{0}$ u.s.c., $g_{1}$ continuous, $g_{0} \leqq g_{1}, g_{0}=g_{1}$ in $\operatorname{fr}_{Y} F$. Let $A$ be closed and $U, W$ open in $X \times Y \times R$, and suppose (o being a basepoint in $X$ ) that

(1) $o \times \Pi\left(g_{0}, g_{1} \mid F\right) \subseteq W$,

(2) $A \cup\left(o \times \Pi\left(g_{0} \mid F\right)\right) \subseteq U$,

(3) $A \cap\left(o \times \Pi\left(g_{0}, g_{1} \mid F\right)\right) \subseteq o \times \Pi\left(g_{0} \mid F\right)$.

Then $A \cup\left(o \times \Pi\left(g_{0}, g_{1} \mid F\right)\right)$ can be engulfed by $h U$, where $h$ is active only in $W$.

Theorem $\mathrm{A}$ is proved by elementary point-set topology.

\section{REFERENCES}

1. J. Stallings, Polyhedral homotopy spheres, Bull. Amer. Math. Soc. 66 (1960), 485.

2. - The piecewise linear structure of Euclidean space, Proc. Cambridge Philos. Soc. 58 (1962), 481.

3. E. C. Zeeman, Seminar on combinatorial topology, Institut Hautes Etudes Sci. Publ. Math., Paris, 1963-1965.

UNIVERSITY OF WISCONSIN AND RICE UNIVERSITY 